JASEM ISSN 1119-8362

All riqhts reserved
Available Online at http:// www.bioline.org.br/ja
J. Appl. Sci. Environ. Mgt. 2004

Vol. 8 (2) 67 - 69

\title{
Chlamydia trachomatis serovars of endemic trachoma had been predominantly existed in Japan?
}

\author{
Kei Numazaki, \\ Department of Pediatrics \\ Sapporo Medical University School of Medicine \\ S.1 W.16 Chuo-ku, Sapporo, 060-8543 Japan \\ TEL: (011) 611-2111 EXT.3413 \\ FAX: (011) 611-0352 \\ E-mail: numazaki@sapmed.ac.jp
}

\begin{abstract}
In contrast to urogenital chlamydial infection, trachoma is a household disease that has disappeared in Japan as many parts of world because of improved living conditions and hygiene. However, chlamydial inclusion conjunctivitis is still not a rare disease in the outpatient clinics of general Japanese ophthalmologists. The serovars that we identified from Japanese infants and pregnant women were similar to those reported in other studies from non-trachoma-endemic areas and were thought to be mainly urogenital tract-origin. Once Japan was thought to be belong to an endemic area of trachoma as other Asian countries. Chlamydia trachomatis serovars of trachoma-endemic area had been really existed and predominantly associated with trachoma in Japan? @JASEM
\end{abstract}

Trachoma, a chronic keratoconjunctivitis, is still recognized as one of most important origins of blindness in developing countries and inclusion conjunctivitis of both adults and newborns is associated with sexually transmitted diseases (STD) in developed countries. The "SAFE" strategy is used for the control of trachoma: surgery for in-turned lashes, antibiotics for active disease, facial cleanliness, and environmental improvement (Mabey et al., 2003). By means of the SAFE strategy, WHO and its partners aim to eliminate trachoma as a public-health problem by the year 2020 .

Genital infections caused by $C$. trachomatis are the most common STD and are recognized as one of the most important public health problems in many industrialized countries including Japan.

In contrast to urogenital chlamydial infection, trachoma is a household disease that has disappeared in Japan as many parts of world because of improved living conditions and hygiene. Acute inclusion conjunctivitis in adults is known as follicular conjunctivitis associated with epithelial keratitis and small degree of corneal vascularization. Chlamydial inclusion conjunctivitis is still not a rare disease in the outpatient clinics of general Japanese ophthalmologists (Aoki et al., 1998; Isobe et al., 1996).

\section{MATERIALS AND METHODS}

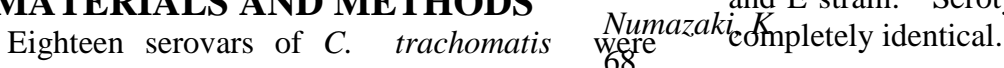
classified by the microimmunofluorescence (MIF) test (Wang and Grayston, 1974). The epitopes that distinguish serovars reside principally on major outer membrane protein (MOMP). The sequences of the MOMP gene which includes four variable domains (VDs) have been determined for all 15 serovars.

We used the polymerase chain reaction (PCR) to amplify a large part of the MOMP gene (Omp1), including four VDs, and then cataloged restriction fragment length polymorphism (RFLP) to determine the serotypes of clinical specimens from genotypes of $C$. trachomatis (Numazaki et al., 1998; Ikehata et al., 2000).

\section{RESULTS AND DISCUSSIONS}

Nasopharyngeal and conjunctival specimens were collected from Japanese infants with pneumonia, from 1 day to 1 year old. Endocervical specimens were obtained at the time of 28 to 30 weeks of gestational age from asymptomatic Japanese pregnant women.

Conjunctival swabs were also collected from Japanese adult pairs of sexual partners in 1day- to 28-year-old age with acute follicular conjunctivitis.

The typing of infantile nasopharyngeal and conjunctival specimens gave us the following results: $\mathrm{D}, \mathrm{E}, \mathrm{F}, \mathrm{H}$, and variants of serovar $\mathrm{D}$. The typing of endocervical specimens from asymptomatic Japanese pregnant women also gave us the following results: D, E, F, G, H I, J, K, $\mathrm{D}$-variants and variants of endemic-trachoma serovar B. The typing of adult conjunctival specimens gave us the following results: $G, D$ and $\mathrm{E}$ strain. Serotypes between each pair were

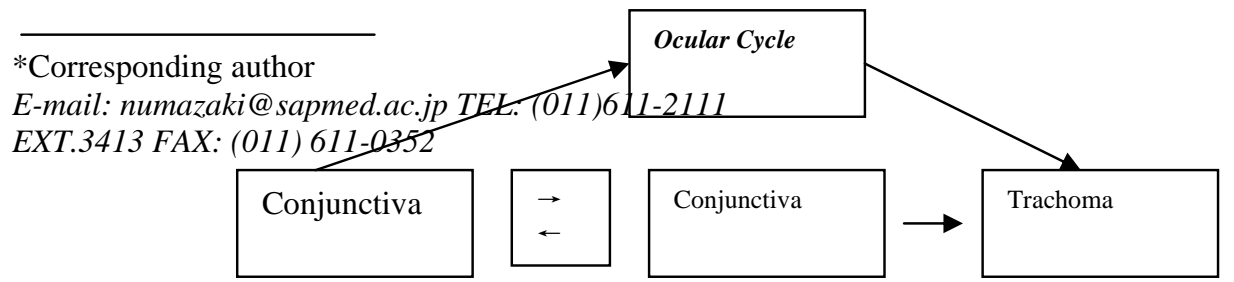


C. trachomatis serovars of $\mathrm{A}, \mathrm{B}, \mathrm{Ba}$ and $\mathrm{C}$ have been predominantly associated with endemic trachoma. The serovars $\mathrm{D}$ though $\mathrm{K}$ generally been isolated from the urogenital tract. However, any serovar of $C$. trachomatis including urogenital tract-origin can cause inclusion conjunctivitis and the clinical manifestations of trachoma are thought to be due to the complex pathogen-host relationship in disease. Presence of ocular and urogenital cycles of $C$. trachomatis infections were speculated (Figure 1). In trachoma-endemic areas, severe disease leading to scarring and blindness may be the result of frequent reinfection of different serovars of $C$. trachomatis including extraocular and urogenital tract-origin.

The serovars that we identified from Japanese infants and pregnant women were similar to those reported in other studies from non-trachoma-endemic areas and were thought to be mainly urogenital tract-origin (Wagenvoort et al., 1988). Similar results were also obtained from the study of adult inclusion conjunctivitis in Japan (Isobe et al., 1996). Even some of serovars originally associated with endemic trachoma are occasionally detected in urogenital infection. Chlamydial pneumonia of Japanese infants were speculated to be mainly caused by mother-to-infant transvaginal transmission of $C$. trachomatis. Antigenic variations of $C$. trachomatis were found among the strains from nasopharyngeal, conjunctival and endocervical origins.

Once Japan was thought to be belonging to an endemic area of trachoma as other Asian countries. This endemic trachoma had been thought to be brought from China to Japan. However, the serovars of $C$. trachomatis from patients with trachoma at that time had been unclear. $C$. trachomatis serovars of trachoma-endemic area, such as A, B, Ba and C, had been really existed and predominantly associated with trachoma in Japan? If these serovars had existed, why serovars of endemic trachoma disappeared and serovars of urogenital tract-origin became predominant? Characterization of unclassified variants will allow more detailed epidemiological studies of C. trachomatis infections in Japan. 


\section{REFERENCES}

Aoki K, Sato C, Hashimoto N, Chiba S, Numazaki K (1998). Clinical and etiological studies of chlamydial conjunctivitis in Sapporo, Japan. Jpn J Ophthalmol 32: 444-449.

Ikehata M, Numazaki K, Chiba S (2000). Analysis of Chlamydia trachomatis serovars in endocervical specimens derived from pregnant Japanese women. FEMS Immunol Med Microbiol 27: 35-41.

Isobe K, Aoki K, Itoh N, Ohno S, Takashima I, Hashimoto N (1996). Serotyping of Chlamydia trachomatis from inclusion conjunctivitis by polymerase chain reaction and restriction fragment length polymorphism analysis. Jpn $J$ Ophthalmol 40: 279-285.
Numazaki K, Ikehata M, Chiba S, Suzuki K, Hashimoto N (1998). Unclassified serovars of Chlamydia trachomatis isolated from Japanese infants. Clin Microbiol Infect 4: 519-523.

Wagenvoort JHT, Suchland RJ, Stamm WE (1988). Serovar distribution of urogenital Chlamydia trachomatis strains in the Netherlands. Genitourin Med 64: 159-161.

Wang S-P, Grayston JT (1974). Human serology in Chlamydia trachomatis infection with microimmunofluorescence. J Infect Dis 130: 388-397.

Mabey DC, Solomon AW, Foster A (2003).

Trachoma. Lancet 362: 223-229. 